\title{
DUE PERCORSI AUTOBIOGRAFICI FRA I TESTI DI LUDOVICA RIPA DI MEANA
}

TWO AUTOBIOGRAPHIES FROM LUDOVICA RIPA DI MEANA'S WORKS

Andrea Casoli

Universidad de Bolonia, Italia

\section{Riassunto:}

Nei romanzi e nei testi teatrali di Ludovica Ripa di Meana la dimensione autobiografica risulta quasi completamente assente. ऐ̀ rintracciabile, in forme originali nel librointervista al filologo Gianfranco Cortini e nel suo primo testo, Asma. Attraverso questi due testi emerge come il tema autobiografico sia uno strumento fondamentale alla ricerca della propria voce.

\section{Parole chiave:}

autobiografico, alterità, corpo, voce, autore

\section{Abstract:}

In the novels and theatrical texts of Ludovica Ripa diMeana the autobiographical dimension is almost completely absent. It can be found in original forms in the book-interview with the philologist Gianfranco Cortini and in his first text, Asma. Through these two texts it emerges that the autobiographical theme is a fundamental tool in the search for one's own voice.

\section{KEYWORDS:}

autbiographical, otherness, body, voice, author 


\section{Autobiografico “Altro": Diligenza e voluttà}

Se si eccettua il primo romanzo, La sorella dell' Ave (e, a voler essere puntuali fino all'intransigenza, solo la prima parte di quello), l'opera di Ludovica Ripa di Meana appare sostanzialmente estranea alla dimensione autobiografica. Ulteriore eccezione all'eccezione, il testo Asma, che avrebbe costituito, se fosse approdato alla pubblicazione, l'esordio alla scrittura autoriale piena di Ludovica Ripa di Meana; trattandosi però di un testo rimasto inedito, mi piace l'idea di riservargli uno spazio a sé, quasi appendice e variazione sul tema dell'autobiografico: da "altro" a "privato", come vedremo; sia perché cronologicamente l'autobiografico altro costituisce un'esperienza precedente e maggiormente articolata rispetto a quello privato; sia perché il fatto che il secondo sia rimasto inedito, mentre il primo ha ottenuto notevoli riscontri di popolarità, è elemento cui riservare attenzione, cui attribuire un particolare significato. ${ }^{1}$ Sotto l'etichetta di «autobiografico altro» si possono annoverare tre libri pubblicati a opera di Ludovica Ripa di Meana, da leggersi come la punta di un iceberg, il risultato più maturo di una lunga frequentazione dell'ambiente letterario e giornalistico, teatrale e televisivo, l'esito finale di una carriera fitta d'interviste: Il paradiso è un cavallo bianco che non suda mai per Adriano Celentano, ${ }^{2}$ Dietro l'immagine. Conversazioni sull'arte di leggere l'arte per Federico Zeri ${ }^{3}$ e Diligenza e voluttà con Gianfranco Contini. ${ }^{4}$ Accanto a essi, distinta solo per il mezzo a cui è destinata, la storica intervista televisiva a Carlo Emilio Gadda: Sulla scena della vita, realizzata con Gian Carlo Roscioni e trasmessa dalla Rai il 5 maggio $1972 .^{5}$

Questi quattro lavori sono accomunati, a vario titolo, da quella sorta di casualità che governa il mestiere del giornalista culturale: incontri, anniversari, pubblicazioni, opportunità commerciali intorno a cui sorge un'intervista che può far da scintilla allo sviluppo di un dialogo più ampio e articolato e dunque anche dar luogo a un progetto editoriale. Nell'eterogeneità che segna i titoli raggruppati sotto l'etichetta di «autobiografico altro» appare evidente per varie ragioni che l'analisi del libro intervista a Gianfranco Contini risulta quella più interessante e stimolante. Innanzi

1 Un'appendice all'eccezione, senza che la regola enunciata possa esserne sminuita, è costituita poi dalle poesie: raccolte sotto il titolo Rime e tuttora inedite.

2 Adriano Celentano, Il paradiso è un cavallo bianco che non suda mai, Sperling \& Kupfer, Milano 1982.

3 Federico Zeri, Dietro l'immagine. Conversazioni sull'arte di leggere l'arte, Longanesi, Milano 1987.

4 Ludovica Ripa di Meana - Gianfranco Contini, Diligenza e voluttà. Ludovica Ripa di Meana interroga Gianfranco Contini, Longanesi, Milano 1989.

5 Una versione ridotta dell'intervista è stata edita in videocassetta nel documentario Gadda racconta Gadda, a cura di Mauro Bersani e Maria Paola Orlandini, regia di Antonella Zecchini, Rai Educational, 2003 allegato al volume di Mauro Bersani, Gadda. La vita e le opere, Einaudi, Torino 2003. 
tutto per l'appartenenza al comune ambito letterario, che porta molte delle domande di Ludovica Ripa di Meana a concentrarsi intorno ai temi della lettura e della scrittura, della critica militante e dell'erudizione e, nell'insieme, dell'universo letterario nei suoi molteplici aspetti. Poi c'è il fatto che, a differenza dei precedenti, in quest'ultimo testo non c'è una mimesi della voce dell'altro, ma la messa in scena del dialogo con l'altro, che costringe l'intervistatrice a farsi astante, attrice, a scoprirsi maggiormente.

Ancora, seppur in apparenza motivo meno rilevante, la mia predilezione per l'analisi di questo libro a discapito degli altri, oltre che per l'ovvia affinità della materia trattata, è supportata dalla minor casualità nella scelta del "personaggio", e dalla maggior difficoltà dell'operazione sia compositiva sia editoriale. Mentre il libro per Celentano o quello per Zeri erano fin dal principio destinati a un buon successo commerciale, mentre l'intervista in limine mortis a Carlo Emilio Gadda conteneva già, per l'eccezionalità della situazione, le caratteristiche dell'evento storico, il libro intervista a Gianfranco Contini si configura fin dal concepimento come scommessa, come sfida, come una vera e propria impresa autoriale. Se per gli altri tre lavori è facile ipotizzare un buon ritorno economico fin dall'origine della scelta e del progetto, per quest'ultimo è vero invece il contrario.

Se per Federico Zeri e per Adriano Celentano si trattava di trasporre sulla pagina il fascino e il segreto della voce e della personalità dell'estroso critico d'arte o delpiù popolare cantautore italiano; se per intervistare Carlo Emilio Gadda si trattavadi vincerne la proverbiale ritrosia, insinuandosi affettuosamente fra le sue nevrosi; il libro di Contini è invece tutto da inventare: il libro di Contini è tutto un'invenzione. Prova ne sia che il risultato è un unicum nel panorama editoriale non solo italiano. ${ }^{6} \mathrm{La}$ prima invenzione è proprio questo libro in sé, l'idea che possa esserci il ritratto di unodei massimi esponenti della filologia, di un mostro sacro dello studio e della critica chetutto è stato fuorché un personaggio. La prima invenzione, che fa di questo un libro da accostare ai romanzi e alle tragedie di Ludovica Ripa di Meana, è il personaggio di Gianfranco Contini.

Il secondo azzardo, non certo minore, è quello di aver definito questo libro «la prima autobiografia di un grande intellettuale italiano del Novecento», chiarendo che la matrice autoriale risiede all'interno di una speciale e irripetibile dimensione dialogica paritaria, in cui l'intervistatrice e l'intervistato, le domande e le risposte concorrono nella medesima proporzione alla costruzione della voce narrante. Nel testo del risvolto (con amplissima probabilità dovuto, come d'abitudine, alla mano del marito,

$6 \quad$ All'interno di questo assai poco frequentato genere editoriale, va segnalato un altro libro di eccezionale valore: Camminare nel tempo di Ezio Raimondi (Aliberti, Reggio Emilia 2006), realizzato in dialogo con due allievi del grande italianista, Alberto Bertoni e Giorgio Zanetti. La natura degli interlocutori e la dimensione del dialogo fra gli allievi e il maestro danno luogo a un libro di tutt'altra atmosfera, con una dimensione autoriale completamente differente. 
Vittorio Sermonti) si legge che sono «la disponibilità integrale alle storie e alle voci degli interlocutori» e insieme «un'aristocratica assenza di protagonismo»a consentire a Ludovica Ripa di Meana di «conversare alla pari» con persone così autorevoli nei rispettivi campi.

Il concepimento e la nascita di questo libro si colloca infatti «nello spazio che intercorre fra due persone così remote per origine, indole e applicazione al mestiere di esistere». $\mathrm{E}$ in questo senso, la dimensione culturalmente «impregiudicata» di Ludovica Ripa di Meana, armata solo di «una passione bruciante e disordinata per i libri», produce un dialogo sorprendente, coraggioso, improntato alla curiosità e alla libertà, un dialogo che si può arrivare a definire "romanzesco". Non solo nuovo, ma anche e soprattutto "altro".

Il fatto che a intervistare l'autorevolissimo filologo e l'influente critico militante, l'intellettuale che, esprimendosi ai massimi livelli in entrambe le discipline, ha rappresentato un modello ineguagliato, ${ }^{7}$ non sia uno dei suoi allievi bensì una giornalista, induce la conversazione a concentrarsi anche su aspetti personali, solo apparentemente minori, che giusto l'irriverenza di un rapporto libero e paritario ha potuto far emergere.

E non va nemmeno trascurato l'apporto fondamentale dell'elemento femminile alla costruzione di questa voce autoriale, se è vero, come sostiene Mercedes Arriaga Flórez, che «il carattere detotalizzante dell'autobiografico femminile è strettamente legato al rapporto che la scrittura instaura sia con la "materialità" della vita quotidiana, sia con la "materialità" del corpo». ${ }^{8}$ Basterebbe per la prima vedere con che immediatezza e spontaneità l'intervistatrice riesce a domandare a uno dei massimi studiosi del suo tempo, noto per austerità e ritrosia, se sia goloso e se preferisca la polenta sciolta o quella granulosa, cosa significhi per lui darsi del lei oppure darsi del tu, se amasse i Beatles, se abbia paura dell'aeroplano e altri simili dettagli che finiscono per restituire al lettore molte importanti informazioni capaci non solo di rivelare l'uomo oltre la cortina di libri dei suoi studi, ma anche di proiettare su questi ultimi una luce inedita e rivelatrice. Per la seconda, invece, è sufficiente notare come ciascuna delle rievocazioni di amici o personaggi di rilievo del mondo culturale con cui Gianfranco Contini è

7 Nella vastissima bibliografia continiana si possono eleggere a emblemi di questa duplice passione, da una parte il classico Un'idea di Dante, Einaudi, Torino1970, la cura delle Rime, Einaudi, Torino 1939, Il Fiore e il Detto d'amore attribuibili a Dante Alighieri nell'edizione nazionale, Mondadori, Milano 1984 e l'antologia Poeti del Duecento, Ricciardi, Napoli-Milano 1960; dall'altra la serie Esercizî di lettura, Einaudi, Torino 1974, Ultimi esercizî ed elzeviri, Einaudi, Torino, 1987, Postremi esercizî ed elzeviri, Einaudi, Torino 1998 e i volumi Una lunga fedeltà. Scritti su Eugenio Montale, Einaudi, Torino 1974 e Quarant'anni d'amicizia. Scritti su Carlo Emilio Gadda, Einaudi, Torino 1989.

8 Mercedes Arriaga Flórez, Mio amore, mio giudice. Alterità autobiografica femminile, Manni, Lecce 1997, pagina 9. 
entrato in contatto, contenga delle costanti e precise, a volte sintetiche e fulminanti, notazioni sull'aspetto fisico, sulla loro gestualità. Di Giorgio Morandi Contini dice:

Lo conobbi subito dopo l'ultima guerra, grazie al comune amico Giuseppe Raimondi (con cui poi, purtroppo, ruppe). Era un uomo fisicamente straordinario, che nella sua casa di via Fondazza, ammobiliata e arredata con solidità ottocentesca, allevava compendî di umili stoviglie in cui era metafisicamente concentrata la virtù antica, che veniva variando nei suoi «dipinti» all'infinito. Altissimo, ossuto, con una frangetta canuta, sdentato, nuotante in abiti troppo larghi, era allora semplice, ascetico, ingenuo nel senso pristino della parola. Più tardi venne in lui insinuandosi la diffidenza, ma preferisco rammentarlo nella sua fase di eremita cordiale. Che sia il maggior pittore italiano di questo secolo, per me non fa dubbio.

\section{Di Filippo De Pisis, Contini dice:}

Sono stato amico di De Pisis, uomo finissimo e di grande bontà sotto la specie di un corpo triviale e vizioso. L'ho frequentato molto a Parigi, e ho visto man mano mutare la sua maniera di fa-presto in modi interessantissimi cui forse non è stata data la debita attenzione. Diceva di essere un discreto pittore ma soprattutto un poeta, e forse un poco ci credeva.

\section{E su Benedetto Croce:}

Ma com'era Croce, personalmente? Era simpatico? Affasci-nante? era disponibile? umanamente avventuroso?

Singolare sfilata di aggettivi, se permette, alieni in tutto da Croce. Simpatico, certo, era, verso gli amici almeno, ma dominato da quella che in buon italiano si chiamava «sprezzatura». Era ospitale senza ostentazione; e trasandato nel vestire, ma come un «galantuomo» delle sue parti. Il suo discorso era disseminato di epitafî culturali (per esempio: «la Germania, figlia primogenita dell'Europa»), ma la trama generale era aneddotica. Amava fisicamente i libri. I suoi, tranne che in un'aula centrale a due piani, erano tutti ad altezza d'uomo, e di uomo non alto. [...] Non ricordo di averlo mai visto ridere, né sorridere, nonostante i sali fescennini che seminava, rilevati dall'accento fortemente vernacolare, che smottava spesso nel dialetto.

Tutti questi elementi depongono a favore di una scrittura autobiografica che vuole essere vissuta non in antitesi alla scrittura romanzesca, bensì come figura, tropo che attraversa i generi letterari. E in questa direzione, ancor più suggestiva e prolifica si rivela l'intuizione di Paul De Man, ${ }^{9}$ secondo cui l'autobiografia più che un genere letterario vero e proprio è una figura di lettura, portando a compimento per certi versi

99 Paul De Man, "La autobiografía como desfiguración", in La autobiografía y sus problemas teóricos, Angel G. Louriero (a cura di), in «Suplementos Anthropos», n. 29, Anthropos, Barcelona 1991, pagine 113-117; e, soprattutto, il suo Allegorie della lettura, Einaudi, Torino 1997. Sul rilievo delle teorie di De Man sono fondamentali Jacques Derrida, Memorie per Paul de Man, Jaca 
e per tutt'altre vie le riflessioni bachtiniane sulla vita storica dei generi letterari che si realizza e giunge a vera pienezza solo nella modulazione definita dalla partecipazione dei lettori. ${ }^{10}$ Una dimensione nuova dell'autobiografico appartiene in generale a questa tipologia di scrittura, a prescindere dall'originalità e dalla singolarità del caso in oggetto. Il contesto generale del secondo Novecento, infatti, segnato com'è dalla diffusione delle nuove tecniche di comunicazione, ha prodotto una sorta di espansione dell'universo autobiografico e una nuova modulazione dei rapporti fra i generi letterari che lo attraversano, proprio a partire dai nuovi media (la radio, il cinema e la televisione) in cui può trovare espressione. ${ }^{11}$ Il segno della riuscita fusione delle due parti, quella interrogante e quella interrogata, in una voce narrante come unico protagonista della scena è qui ulteriore prova, sotto un aspetto in vero assai particolare più legato alla forma che non al contenuto, del paradosso tipico dell'autobiografia, che la vuole finzione per chi la scrive e verità per chi la legge. ${ }^{12} \mathrm{E}$ in questo caso, a maggior ragione, tornano precise e rivelatrici le analisi bachtiniane del rapporto tra autore e personaggio, che riproduce il rapporto tra parola letteraria e vita:

L'autore deve situarsi fuori di sé, vivere se stesso su un piano diverso da quello su cui noi effettivamente viviamo la nostra vita; soltanto a questa condizione egli può integrare se stesso, fino a diventare una totalità [...]; egli deve diventare un altro rispetto a se stesso, guardarsi con gli occhi di un altro. ${ }^{13}$

La dimensione romanzesca di questo libro, il suo carattere estetico, per restare all'interno del binomio bachtiniano, sono sanciti dall'invisibilità dell'autore che «indossa la veste del tacere», si rende invisibile, non è più un io personale, soggettivo, bensì un io testuale, diviene principio e origine che dà coerenza e unità al testo secondo la celebre nozione foucaultiana. ${ }^{14}$ La voce narrante è una sola, frutto delle domande e delle risposte che si integrano in un dettato coerente, compatto, pur rimanendo sulla scena due astanti, due attori di cui si perde la distinzione di quale sia indispensabile all'altro, un po' come in quelle coppie in cui non è possibile immaginare il comico senza la sua spalla o in cui addirittura si perde la distinzione tra l'uno e l'altro ruolo.

Book, Milano 1995, Guido Guglielmi, Paul de Man e le aporie della lettura, in Id., La parola del testo. Letteratura come storia, il Mulino, Bologna 1993, pagine 121-151, Eduardo Saccone, Pratica e teoria della lettura (1997), introduzione a Paul de Man, Allegorie della lettura, cit., e Francesco Longo, Paul de Man. La lettura retorica, Aracne, Roma 2008.

10 Michail Bachtin, Estetica e romanzo, Einaudi, Torino 1979.

11 Marziano Guglielminetti, "Biografia e autobiografia", in Letteratura italiana, V: Le Questioni, Einaudi, Torino 1986, pagine 829-886.

12 Dario Villanueva, "Realidad y ficción: la paradoja de la autobiografía”, in Escritura autobiográfica, José Romera Castillo e altri (a cura di), Actas del seminario internacional del Instituto de Semiótica literaria y teatral, Madrid, UNED, 1-3 luglio 1992, Visor, Madrid 1992, pagine 15-33.

13 Michail Bachtin, Estetica e romanzo, cit., pagina 15.

14 Michel Foucault, L'ordine del discorso, Einaudi, Torino 1972. 
Al punto che, forse forzando un po' i termini del discorso, ma poi neanche tanto, a dire il vero, è possibile leggere in controluce di queste parole in cui Contini parla della moglie, alcuni tratti che caratterizzano il profilo culturale di Ludovica Ripa di Meana, soprattutto per quel che riguarda la definizione di «lettrice impregiudicata»:

[Maragaret] è stata mia allieva, diciamo, tre settimane, e in un campo di vacanze in Austria, quindi il rapporto è stato, allora, veramente unilaterale. Però da quando ci siamo sposati, e anche da prima, ho imparato moltissimo da lei. Intanto perché è di un'altra lingua, e poi perché maneggia quattro lingue, e perché è una lettrice assolutamente impre-giudicata... mi ha insegnato moltissime cose. Qualcuna può averla imparata da me, ma sono certissimo che il bilancio è squilibrato: sono io che ho imparato molto di più da lei. E séguito. E mi piace discorrere con lei. Forse non di argomenti tecnici, ma di cose letterarie sì. Cosa vuol dire «è una lettrice impregiudicata?» Che non ha pregiudizi; che per giudicare se un libro è bello, non ha bisogno che glielo sia cantato da altri; e che se un libro è famoso, non per questo lo giudicherà un capolavoro. No, è assolutamente libera da pregiudizi. E quindi ha una libertà di giudizio della quale ci si può fidare.»

Il modo più semplice e immediato per dimostrare quanto questo libro-intervista, che si presenta come una serie di domande e risposte, senza alcuna didascalia o nota o preambolo, suddiviso in capitoli tematici, risulti nel suo insieme davvero romanzesco è forse quello di ripercorrerne i momenti più significativi. Si prenda l'incipit con quel suo movimento circolare, tipico di molti testi di Ludovica Ripa di Meana, istintivamente votati a evitare una linearità semplificatrice, falsamente onesta, che illuda di una possibile chiarezza definitiva:

Io vorrei dire che sto finendo dove incominciai, e il circolo si chiude...

Per me è un vero onore ascoltare Gianfranco Contini, e anche un'avventura. Spero di essere

all'altezza. Da dove cominciamo, professore?

Mah... da dove lei vuole.

Proviamo a cominciare da quando lei non c'era? Mi racconta dei suoi genitori...

Senta: di un famoso professore si diceva che proclamasse di essere stato benedetto nei figli. Io sono stato benedetto nei genitori...

O si prenda, un capitolo esemplare dell'intero testo, per provare a delinearne alcune peculiarità: quello in cui Ludovica Ripa di Meana interroga Gianfranco Contini a proposito di un altro gigante della cultura italiana del Novecento, il critico d'arte Roberto Longhi. In questo capitolo, intitolato Soprattutto un profilo, l'autore conduce il lettore nella propria officina, per certi versi mostrando come armeggia con i ferri del mestiere di scrittore, in un gioco di rimandi in cui Ludovica Ripa di Meana ritrae Gianfranco Contini mentre ritrae Roberto Longhi:

Un ritratto di Longhi non è facile da tracciare, ma l'ingrediente importante è quello della sua semplicità. Questo uomo che pareva scostante, remoto e difficile, in sostanza 
era un uomo autentico, semplice e schietto. Lo accusavano di fare poche lezioni. Io ne seguii alcune, ma quelle che faceva, certo, erano incantevoli. Non erano lezioni impressionanti: erano di una schiettezza estrema, ma rivelatrici di fatti nuovi. Non parlava mai di cose che non fossero o sicuramente collaudate o scoperte. Certo, è un grande scrittore, che passò attraverso molte fasi; e credo che abbia ragione Cecchi quando rileva che la sua ultima fase fu la fase classica, visto che da giovane era stato un settatore dei futuristi, poi venne evolvendo verso una sua «maniera», e poi la maniera evolse in qualcosa di estremamente puro, limpido. Insomma, direi che le sue ultime cose - il Caravaggio, per esempio - sono degli scritti di una limpidità sovrana.

E poi, come rilevato anche in precedenza, la costante della materialità del corpo ritorna quale elemento imprescindibile nella costruzione della memoria dell'amico, e del suo carattere:

E come se lo ricorda, anche fisicamente... il primo ricordo, diciamo... Come le sembrò quel signore, quell'uomo, quella persona seduta o in piedi?

Mi parve soprattutto un profilo: un profilo imperioso e sdegnoso, che poi celava una grande bontà e disponibilità all'amicizia nonostante le apparenze, le soprastrutture... Era un grande attore, voglio dire.

Un grande attore? Certo. Lui voleva veramente essere attore, avrebbe voluto fare l'attore, e in realtà riuscì a realizzare questa sua vocazione di mimo. Era un mimo pungente. Non soltanto come imitatore, ma completamente, come rappresentante di un perso-naggio, ecco... gestiva il suo personaggio. Un personaggio che era qualche volta elegantissimo e qualche volta estremamente trascurato. Perché Longhi voleva mettere tra sé e gli altri il personaggio? Bisognerebbe essere attori per saperlo. Credo che ogni attore metta tra sé e gli altri un personaggio... Cosa che non gli impediva di essere lui, e di lasciarsi raggiungere. Ecco, la cosa rara per un personaggio che era attore, è che si lasciava raggiungere dagli amici. Ma quella chiavicina dell'amicizia era molto difficile da ottenere. Del resto era molto ricco di umori e spesso non si poteva andar d'accordo. Quando avvertivo che le sue opinioni divergevano dalle mie, e io non potevo tacerle, per qualche tempo, così, rallentavo la frequentazione, poi ritornavo... e quindi riuscii a salvare sempre la nostra amicizia. [...]

Quel misto di spavento e di deferenza che Longhi incuteva a chi non aveva la ventura di essergli amico... Longhi lo faceva anche per ironia, di mettere un po' di distanza tra sé e gli altri?

Ma l'ironia era molto visibile, però non ritengo che fosse universale. Cioè, l'ironia si mescolava - e non tutti lo avevano capito - a una certa ingenuità, a un certo candore. Era ironico e candido. E non so se sapesse di essere candido. 
Era questo il suo lato infantile? Certo, certo. Molto molto importante. Molto importante per spiegarlo.

Per spiegare anche il suo talento? Certo.

... e la sua grandezza critica? Certo, certo.

E la libertà con cui Ludovica Ripa di Meana provoca Gianfranco Contini a svestire i panni dell'austero filologo per fargli raccontare una "barzelletta" su Longhi:

Senta, e delle storie belle di Longhi? mitiche... o esilaranti... Oh, Dio. Non so. Ma non vorrei ridurre Longhi sul piano della barzelletta... perché, per la verità, lui inclinava, inclinava con una certa enfasi alla barzelletta... Gliene do una: mia moglie è westfalica, no?, e lui diceva: «Gianfranco ha trovato moglie in Westphalia! L'ha vista in West-phalia, e se n'è innamorato!». È un tipico esempio delle sue facezie.

E di nuovo la materialità, questa volta l'attenzione cade sulla materialità dello sguardo, approssimandosi al segreto del magistero critico di Roberto Longhi:

E quando lui guardava un quadro, magari un quadro che non aveva mai visto, succedeva qualcosa nella sua fisionomia? Socchiudeva gli occhi. Socchiudeva gli occhi e guardava un minimo particolare. Era dal particolare che risaliva all'universale. Tanto è vero che agli studenti faceva fare dei quiz. Prendeva, mettiamo, un pezzettino di un quadro, non so, di Mantegna o di Caravaggio, e lo faceva riconoscere. Ricordo che fece questo esamino a mio figlio Roberto, quando era bambino, avrà avuto sette o otto anni, e Roberto lo passò... Passò l'esame con Longhi!

Ma subito a questo si accosta il dettaglio quotidiano, minimo, che risponde a una curiosità piccola e sincera, che rivela al lettore il privato, l'uomo, dietro l'immagine pubblica del filologo:

A proposito del guardare, professore, come mai lei non porta gli occhiali? Ogni tanto, in certe fasi della mia vita... portavo gli occhiali per guardare i quadri. Ci fu il grande periodo delle mostre, soprattutto dopo la guerra, specialmente in Svizzera. Ricordo di avere usato degli occhiali.

Però è abbastanza eccezionale che alla sua età lei non usi gli occhiali facendo il lavoro che fa...Sì, ma vede, dall'occhio sinistro sono miope, dall'occhio destro sono ipermetrope, e i miei compagni di medicina, in collegio, mi profetavano che a ventotto anni (non so perché proprio a ventotto!) sarei diventato strabico. È passato un certo numero di anni, ma strabiconon sono diventato. Io leggo col sinistro e vedo in lontananza col destro, e questa bigamia, vorrei dire, oculare, mi funziona perfettamente. 
Longhi, invece, li portava, gli occhiali? Certo, certo... pince-nez portava: i quadri li guardava attraverso il pince-nez.

E usava anche il binocolo? Sì, sì, e la lente. Ma ogni tanto, poi, si toglieva tutto, e aveva lo sguardo del miope, così dilatato, e un po' arrossato. E, allora, parlava con qualche enfasi...

Con grande sapienza, il racconto passa dalla sfera professionale a quella personale, dal mestiere di critico d'arte all'intimità del rapporto con la morte:

Può raccontarmi cosa vuol dire vedere una mostra con Roberto Longhi? In modo particolare una mostra di tesori tedeschi a Berna. E Longhi mi fece vedere come Giotto faceva le aureole. Una cosa assolutamente meravigliosa. Ma, quando lo si accompagnava, diceva ogni tanto qualche parola. Quandogli venival'illuminazione, si fermava e allora parlava. Era veramente straordinario... ma straordinario per la semplicità con cui si metteva alla pari con l'interlocutore. La verità non è su un piano irraggiungibile, la verità è raggiungibile, la verità è umana. Ecco, la sua verità era una verità assolutamente umana, non una verità remota. Forse è anche per questo che la trascendenza gli era del tutto sconosciuta. Il pensiero della morte, credo che non l'abbia mai sfiorato. È l'unico essere umano, io credo, che abbia ignorato la morte.

Non lo interessava? Non lo interessava, no. È una cosa che pochi sanno; e comunque non credo che abbia mai parlato di morte con qualcuno.

Non la temeva? Non esisteva, per lui. Non è riuscito ad accanarla. Per me era un po' stupefacente, anche se me ne sono accorto un po' post factum, visto che io del pensiero della morte sono pieno direi dalla mia infanzia.

Non vorrei, con lei, usare parole troppo improprie, ma quel che mi ha detto riguardava la laicità di Longhi? cioè, lui non aveva il senso...del sacro. Certamente, certamente: era asso-lutamente sprovvisto del senso del sacro.

E questo dipendeva dalla sua educazione? Perché il lavoro che faceva, diciamo, in qualche modo riguarda il sacro... o no? Ma, forse, per evitare queste superfetazioni di falso sacro, lui se lo inibiva completamente. Però, a me sembrava che non fosse una misura igienica... ah, certo, il misticismo percorre la storia dell'arte, il misticismo da lui odiato e, soprattutto, deriso. Mah... in sostanza, ci si sentiva in pace con Longhi. No, Longhi non è che si celasse la morte per una sorta di igiene: era una cosa profondamente spontanea. In qualche modo, se vuole, una cosa aberrante. Che un uomo non pensi alla morte può darsi che sia mostruoso... forse faceva parte del mirabile monstrum che lui era.

E la sua scienza, la sua arte di scrittore e di persona che intuiva e conosceva l'arte degli altri, tutto questo come si mescolava con questa assenza del sacro? Era un 
uomo coi piedi sulla terra, e aveva un senso vivace della realtà. Difatti, in sostanza, le sue grandi scoperte sono scoperte di pittori della realtà. Caravaggio è «pittore della realtà», come dice il titolo della sua famosa mostra.

Infine, a complemento del profilo dell'amico, Gianfranco Contini consegna al lettore un ricordo della moglie di lui, la scrittrice Anna Banti, ancora una volta instradato dalla propria interlocutrice a riscaldare l'immagine pubblica di questi giganti della cultura italiana, con la descrizione degli elementi della loro vita privata, facendone dei "personaggi" a tutto tondo all'interno della narrazione:

E Anna Banti che persona era? Anna Banti era una persona, a prima vista, scostante; aveva, invece, una nascosta bontà che procurava di tacitare, di non mostrare... e che io ho certamente raggiunto. Ma non voleva assolutamente, diciamo, passare per una donna fornita di bontà. Era una donna austera, severa, amara, che custodiva la tranquillità del marito, che si trovava anche in conflitti asperrimi con Roberto, ma che, verso la fine della sua vita, si era adagiata con lui in una amicizia fraterna, in un compagnonnage veramente mirabile. Direi che Longhi aveva riconosciuto le qualità della scrittrice, e le lasciava questa parte. Tanto più, quando lei si occupava di pittori, o di pittrici, com'è il caso, per esempio, dell' Artemisia, o di Lotto. Quando era ancora Lucia Lopresti, fu, oltre il resto, una archivista eccellente, e fece alcune scoperte fondamentali sulla cronologia di Caravaggio.

Questo prima di essere la moglie di Longhi? Prima di essere la moglie, ma essendone l'amica ammirata. È stata sua allieva... e quel corso che ha pubblicato - quel corso che faceva non so se al liceo Ennio Quirino Visconti - quello lo sentì, lei, e si innamorò di quest'uomo, e volle che fosse suo marito. Ci furono delle difficoltà, ma lei riuscì a salvare queste nozze.

Longhi era restìo?... Sì, sì. Era restìo non a lei ma alle nozze in genere, e gli era molto difficile convivere. Difatti convivevano in luoghi separati.

Ah, non vivevano insieme? Non vivevano proprio insieme. Le due camere erano ben distinte e, almeno negli ultimi tempi della loro vita, era una simbiosi molto rispettosa dei diritti dell'altro. Era una donna bella, Anna Banti? Era stata molto bella e aveva mantenuto un portamento regale. Aveva avuto dei capelli rossi di un'attrazione, penso, straordinaria...

E perché aveva fama di essere una donna terribile? Probabilmente perché era molto timida e aveva bisogno di rafforzarsi, di rivaleggiare. Del resto, era una lavoratrice prodigiosa; la sua giornata era diligentemente piena, e Roberto diceva: «Se io avessi la possibilità di scrivere e la tenacia di Lucia, certamente avrei scritto un'opera immensa». Ma mi pare che fosse già sufficiente...

2. Autobiografico privato: Asma 
A seguito di una gravissima crisi asmatica, avvenuta nell'estate del 1988, il marito suggerì a Ludovica Ripa di Meana di provare a raccontare sulla carta la vicenda traumatica appena vissuta, per superare l'impasse psicologica in cui era rimasta bloccata. Leggendo le prime pagine di questa storia, Vittorio Sermonti incoraggiò la moglie a continuare, a scrivere ancora. Tempo di una vacanza e, alla fine dell'estate, quel racconto aveva assunto le dimensioni di un testo di novantanove cartelle dattiloscritte, Asma, tuttora inedito. Un amico che lavorava in una casa editrice, leggendo quelle pagine e apprezzandole, fece notare all'autrice che la sua scrittura non possedeva la cadenza e il passo della prosa, bensì quelli della poesia, e che lei avrebbe dovuto continuare ad assecondare deliberatamente quell'istinto, scrivendo in versi. Non è il caso di attribuire un particolare valore al fatto che il testo non sia stato accolto da quell'editore, che non ne abbia trovato un altro o che l'autrice abbia infine deciso di mantenerlo inedito. Ogni considerazione in merito alle caratteristiche che ne determinarono la mancata pubblicazione, in quella circostanza e forse anche in altre, di cui non ho notizia, non aggiunge molto, anzi direi quasi nulla, al discorso critico su Asma.

È invece un dato significativo il fatto che Ludovica Ripa di Meana abbia messo questo testo nel cassetto definitivamente; sia perché non lo ha più ripreso per correggerlo o modificarlo, sia perché nessuna di quelle pagine e nessuna di quelle storie è stata riutilizzata nei testi scritti successivamente. Agli occhi dei pochi lettori che lo hanno conosciutoAsmasipresentadunquesottol'insegnadella «privatezza»(VittorioSermonti), in termini decisamente radicali. ${ }^{15}$ È l'unico testo autobiografico scritto da Ludovica Ripa di Meana, se si esclude la prima parte del romanzo d'esordio, La sorella dell' Ave, ${ }^{16}$ in cui l'autrice racconta vicende ed episodi che ricalcano quelli della propria infanzia. Un discorso a parte meritano le poesie, che hanno di fatto quasi tutte un carattere fortemente autobiografico, sia quelle legate a elementi della quotidianità, sia quelle legate alla dimensione religiosa, al dialogo con Dio. Questo corpus poetico, ordinato cronologicamente, fino a oggi non è mai stato in alcun modo predisposto e riorganizzato in vista di un'eventuale pubblicazione e, come Asma, è stato relegato a una dimensione privata. ${ }^{17}$

15 Ludovica Ripa di Meana me ne ha fornito copia, così come degli altri testi inediti (la commedia Andiamo, la tragedia in farsa Black in e le poesie scritte dal 1979 a oggi, riunite con il titolo indicativo Rime), in funzione di questa ricerca.

16 Ludovica Ripa di Meana, La sorella dell'Ave, Camunia, Milano 1992.

17 Vittorio Sermonti ha scritto una nota di accompagnamento, a mo' di presentazione di queste inedite Rime, che illustra perfettamente la situazione delle poesie e, per estensione, sotto certi aspetti, anche di Asma: «Se aspettavamo lei... Come abbastanza noto, seppure in ambito abbastanzaangusto, Ludovica ed io viviamo nella stessa casa da un bel pezzo, anche legittimamente coniugati.Che a ciò si debba la mia conoscenza meticolosa di quel che lei scrive ed ha scritto, non lo considero un pettegolezzo. Non mi travesto da talent scout nel ricordare per sommi capi a chi non lo ricordi 
Dunque, mai come in questo caso risultano appropriate le ammonizioni a considerare l'autobiografia come un racconto a sé più che un racconto di sé, a partire dal celebre proposito di Friedrich Nietzsche in Ecce homo: «Mi racconterò la mia vita». ${ }^{18}$

L'affermazione di Jacques Derrida per cui un testo è autobiografico non «perché il firmatario racconta la sua vita [...] ma perché questa vita egli se la racconta, perché egli è il primo, se non il solo, destinatario della narrazione» ${ }^{19}$, in questo caso è doppiamente confermata, sia sul piano concettuale e teorico, sia sul piano concreto e reale. Asma è un testo nato con un intento terapeutico: liberare la propria autrice dall'angoscia e dall'incubo di un nuovo attacco come quello di cui racconta. Questo intento si tramuta quasi subito in pretesto; e al superamento della crisi respiratoria si sovrappone la liberazione della voce dell'autrice: all'interno del testo la ripresa del respiro coincide metaforicamente con la nascita della scrittura.

Asma è un testo difficilmente ascrivibile a un genere letterario, a meno di non volerlo arruolare alla fin troppo folta schiera del romanzo che assorbe tutti i generi, categoria talmente ampia da richiedere poi ulteriori specificazioni per poter essere individuata e utilizzata in termini significativi. Appare invece utile all'intelligenzadel testo consegnarlo all'ambito della scrittura autobiografica, che spesso - specie quella femminile - proprio per la sua natura attraversa i generi e non si pone limiti, ascrivendo come proprio destinatario il proprio autore - un raccontare a sé, come si diceva sopra - e, sarebbe meglio specificare, la propria autrice, appartenendo questi testi, autobiografici e privati, a una tradizione prevalentemente femminile. È questa una scrittura marginale, quando non addirittura emarginata; appartiene a una genealogia dispersa, clandestina, misconosciuta, fitta di testi inediti o scarsamente tradotti: è una parte consistente della scrittura delle donne, quella che ancora, nel suo insieme, stenta ad acquisire un'istituzionalizzazione vera e propria. ${ }^{20}$

Forse allora la vera natura di questo testo è quella del cartone preparatorio e, a uno sguardo critico, Asma appare come un incunabolo della vocazione alla scrittura

- cioè, alle moltitudini dei lettori e alla generalità degli addetti - che la sua bibliografia tardiva include tre romanzi in versi, due tragedie e quattro monologhi (sempre in versi), oltre a un libroconversazione con Gianfranco Contini, ed altre scritture extra-vaganti; alcuni riscontri, spodarici ma piuttosto trionfali (messinscena della tragedia Kouros, e di almeno un monologo; premio Viareggio per la poesia 2003...) non sono bastati ad arruolarla nella "sacra corporazione" dei letterati: leggevo poco fa su un quotidiano famoso un catalogo degli italiani che hanno scritto teatro in versi negli ultimi tempi, e lei non c'era. Insomma, ridottosi l'esercizio della critica militante (salvo rare e benedette eccezioni) alla anamnesi sociologica del successo, è giusto, è bene che Ludovica sia tagliata fuori.

18 Friedrich Nietzsche, Ecce homo. Come si diventa ciò che si è, Adelphi, Milano 1986, pagina 270.

19 Jacques Derrida, Otobiographies. L'insegnamento di Nietzsche e la politica del nome proprio, «Il Poligrafo», Padova 1993, pagine 52-53.

20 Un panorama sia storiografico sia teorico della tradizione della scrittura autobiografica, e nello specifico della scrittura autobio-grafica femminile, è offerto nella prima parte del libro di Mercedes Arriaga Flórez, Mio amore, mio giudice. Alterità autobiografica femminile, Manni, Lecce 1997. 
autoriale di Ludovica Ripa di Meana. A questo si può soprattutto attribuire la variazione dei registri linguistici e dei timbri narrativi che caratterizzano le varie storie che si avvicendano nel testo.

Nelle pagine che raccontano la crisi respiratoria, la narrazione è in prima persona; a prevalere è l'aspetto visionario e allucinato delle descrizioni delle sensazioni e dei pensieri della protagonista. Frasi nominali, ridotte spesso a pochissime, minime parole, tese a segnare il ritmo sincopato del respiro che non c'è e viene invocato con inutili tentativi, si alternano a veri e propri versi, di varia misura, con l'uso in certi casi addirittura della rima, che, innestati nella prosa, conferiscono alla narrazione una dimensione poetica e una musicalità potenti, come ad esempio in questa clausola di sequenza: “... lo sperma della notte l'ha spalmata: è viscida, sfug-gevole, gelata”. Asma merita dunque una particolare attenzione che prescinde dall'idea di un suo possibile recupero editoriale o di un suo mancato inserimento accanto ai testi pubblicati: la sua natura eminentemente autobiografica e privata finisce per essere l'ambito all'interno del quale Ludovica Ripa di Meana si racconta a sé stessa più di quanto non abbia fatto in alcuno degli altri suoi libri.

Che si tratti di un testo rivelatore, di un testo che sancisce la nascita di una scrittrice vera, con una voce personale e con istanze narrative autentiche è provato da diversi elementi che lo caratterizzano. Forse contiene una parte di verità quella provocazione per cui i narratori scrivono sempre solo un libro e tutto il loro mondo, il cuore, è già contenuto nel primo romanzo. Il critico Massimo Onofri, in una recensione,arrivò addirittura a suggerire l'ipotesi di una storia della letteratura attraverso iromanzi d'esordio, sostenendo che tutto quello che si può dire di uno scrittore, nella maggior parte dei casi, è già ricavabile, in nuce, dall'analisi della sua opera prima.In questo caso, questo gioco provocatorio divertente non meno che acuto, andrebbe ancora una volta applicato a metà, essendo Asma il primo testo di Ludovica Ripa di Meana, ma non rappresentando di fatto il suo esordio nel mondo delle lettere. Ciò non dimeno contiene una serie di elementi di sicuro interesse, sia per analizzare la nascita di unavoce autoriale che - non va dimenticato - avviene in età matura, quando Ludovica Ripadi Meana ha cinquantacinque anni; sia perché la destinazione privata sotto la cui luce è stato composto questo testo pone all'evidenza del lettore temi e questioni che nei testi successivi di questa scrittrice appariranno invece dissimulati e assai meno riconoscibili. Considerando che il lettore di queste pagine non ha la possibilità di accedere al testo di cui sto parlando, mi sono risolto ad analizzarlo, seguendone lo svolgimento e avvalendomi di ampi prelievi. Cominciamo dunque dal principio:

Giovedì, 11 agosto, sono andata a Meana per la prima volta in vita mia. E sabato 13 agosto 1988, dopo 13 anni e 13 giorni da quando avevo cominciato,alle 3 del mattino, ho smesso di fumare. Per 40 minuti circa, i bronchi, i polmoni, si sono 
sclerotizzati, asciugati, induriti, fossilizzati, come quei rami di corallo non molato dimenticati nelle vetrine a soffitto alto (l'insegna è semprenera e oro) di qualche gioielleria d'antan, nel nastro tra Napoli e Salerno. Così, e da un istanteall'altro, mentre stavo dormendo un sonno leggero e appetitoso,sgombro, raro sia per l'età che per il mio scassato indotto bronco-polmonare, più irresponsabile di uno scaldabagno a gas. Di colpo mi sono seduta sul letto, come se un rumore mi avesse svegliato. Il petto era di marmo ma credevo di respirare. ${ }^{21}$

La notazione iniziale del viaggio a Meana di Susa, il paese da cui trae origine il nome della famiglia dell'autrice, a pochi chilometri dal confine piemontese con la Francia, si mostra a tutta prima isolata. Apparentemente, un dato consegnato al lettore quasi solo per fornire una contestualizzazione geografica, se si trascura il fatto che l'autrice sta per raccontare della propria minaccia di morte. Al contrario, questo è il primo elemento che ci parla della nascita di una scrittrice e del riconoscimento della propria voce, se seguiamo Roland Barthes quando riconosce nella matrice di ogni racconto il mito di Edipo:

Raccontare non è sempre cercare la propria origine, dire i propri fastidi con la legge, entrare nella dialettica dell'intenerimento e dell'odio?» ${ }^{22}$

La domanda di Edipo, «Perché dunque non dovrei indagare la mia origine?» ${ }^{23}$ rimanda, infatti, la definizione della identità personale all'individuazione delle proprie origini. Adriana Cavarero, in un saggio tra quelli che meglio hanno analizzato dal punto di vista filosofico la natura della narrazione femminile ${ }^{24}$ invita a rivolgersi a Freud piuttosto che a Sofocle, ritenendo che ascrivere il racconto al piacere edipico è un processo che appartiene alla logica fallologocentrica: le storie di vita, invece, ci rivelano in quanto nati da madre. Del mito edipico, invece, vale conservare l'ammonimento all'impossibilità di conoscere la propria storia e alla necessità di sentirla raccontata da altri: la categoria dell'identità personale implica rigorosamente la presenza dell'altro, rimandando all'originaria - materna - dimensione visiva per cui l'identità corrisponde all'esposizione di sé. In una concezione relazionale del mondo per cui, come ha scritto María Zambrano, «tutto è correlato nella vita: il vedere è il correlato dell'esser visto, il parlare dell'ascoltare, il chiedere del dare ${ }^{25}$, Adriana

21 Ludovica Ripa di Meana, Asma, inedito [1989], pagina 1.

22 Roland Barthes, Il piacere del testo, Einaudi, Torino 1975, pagina 46.

23 Sofocle, Edipo re, vv. 1082-1085 in Id., Antigone; Edipo re; Edipo a Colono, a cura di Franco Ferrari, Rizzoli, Milano 1987. Ma anche Edipo: il teatro greco e la cultura europea. Atti del Convegno Internazionale (Urbino 15-19 novembre 1982), a cura di Bruno Gentili e Roberto Pretagostini, Edizioni dell'Ateneo, Roma 1986.

24 Adriana Cavarero, Tu che mi guardi, tu che mi racconti. Filosofia della narrazione, Feltrinelli, Milano 2001.

25 María Zambrano, I beati, Feltrinelli, Milano 1992, pagina 116. 
Cavarero richiama le riflessioni di Hannah Arendt sulla coincidenza ontologica, e non solo fenome-nologica, di essere e apparire:

Il "chi", che appare in modo così chiaro e inconfondibile agli occhi degli altri, rimane nascosto alla persona stessa, come il daimon della religione greca che accompagna ogni uomo per tutta la sua vita, sempre presente dietro le sue spalle e quindi solo visibile a quelli con cui egli ha dei rapporti. ${ }^{26}$

Le storie di vita appaiono dunque prive di autore, persino quando si presentano come autobiografiche, in quanto derivano da un'esistenza che si connota per la sua

«forma relazionale e contestuale dell'esporsi agli altri». ${ }^{27}$ Nell'analisi e nella definizione dell'identità e della soggettività, Adriana Cavarero offre un'immagine del sé relazionale impossibilitato a vedersi e dunque obbligato a esistere nello sguardo altrui, in antitesi al mito di Naciso e al soggetto cartesiano. L'identità del sé, individuale e per questo unica, non deriva da un effetto di discorso, come per gli strutturalisti e i decostruzionisti; è piuttosto un'identità incompleta in sé e dunque proiettata verso l'esterno, tutta volta ad affidare il riconoscimento del proprio senso allo sguardo, ai gesti e alle parole dell'altro. È in questa dimensione che essa si configura come soggetto e oggetto di narrazione.

La filosofia della narrazione di Adriana Cavarero si adatta con proficui risultati all'analisi di alcuni nuclei fondanti dell'opera di Ludovica Ripa di Meana, soprattutto per questo testo iniziale, fondativo e rivelatore, che è intitolato alla morte e ruota interamente intorno alla madre. Il pretesto da cui trae origine la narrazione di Asma è, come rivela il titolo, la minaccia di morte, ovvero il rischio di fronte al quale l'esigenza di definire la propria identità acquisisce una dimensione tangibile e concreta, poiché liminare. Proprio per questo a essere centrale nel testo è il tema della madre, declinato qui in una molteplicità di forme che si ritroveranno parcellizzate e disseminate nelle successive opere narrative e drammaturgiche di Ludovica Ripa di Meana. A riprova di quanto questo tema costituisca un nucleo fondamentale e generativo dell'universo di questa scrittrice, basta osservare come proprio il rapporto con la madre sia l'unico elemento autobiografico presente nella sua opera, e solo e soltanto nella prima parte del primo romanzo.

In principio era la madre. E che si ritorni al principio quando ci s'avvicina alla fine, o per lo meno nel punto di estrema disperazione in cui si intravede la possibilità di essa, è gesto circolare che appartiene non solo a quell' incunabolo della scrittura di Ludovica Ripa di Meana che è Asma, ma anche a molti altri suoi testi, come si verrà dimostrando

26 Hannah Arendt, Vita activa, Bompiani, Milano 1989, pagina 131. Ma si veda anche su questo tema Id., La vita della mente, Il Mulino, Bologna 1987.

27 Cavarero, cit., pagina 52. 
più avanti, in particolare nel capitolo nono, intitolato «Soggetto». Asma risulta, dunque, essere un testo composto di più testi, nel racconto in prima persona della crisi respiratoria s'inseriscono, alternativamente: il monologo rivolto allamadre; la storia in terza persona della «bambina» che è stata l'autrice; pagine di diariodatate all'altezza dell'anno 1962, in cui si narra di una madre (ancora una volta vi si intravede l'autrice) e dei suoi due figli; la Storia di En, altra narrazione in terza persona che recupera episodi mutuati dalla giovinezza dell'autrice; pagine di diario datate all'altezza del 1973, in cui si narra degli ultimi incontri dell'autrice con lo scrittore Carlo Emilio Gadda; un brevissimo atto unico intitolato Lui la mamma e l'altro.

Così elencate, per eterogeneità dei generi edeglistili, queste sequenzedicuisicompone il testo sembrano offrirne un'immagine frammentata, fatta per giustapposizioni, quasi si trattasse di un assemblaggio di materiali preesistenti. Non è così, poiché si possono rintracciare molti richiami tra una sezione e l'altra, scorgere tramature e sviluppi di temi o anche solo di singoli dettagli, che danno compattezza a questo libro sotteso tra i due poli della morte e della madre. Questi due elementi - la morte e la madre richiamano alla mente l'archetipo della Grande Madre, cui si dedicò principalmente uno degli allievi di Jung, Erich Neumann, ${ }^{28}$ appartenente al circolo degli amici di Hannah Arendt a Heidelberg, prima di lasciare la Germania per la Svizzera e approdare infine in Palestina. Già Jung aveva mostrato come il concetto di Grande Madre, proveniente dalla storia delle religioni, abbraccia distinte configurazioni del tipo della dea madre: questo simbolo essendo un evidente derivato dell'archetipo più ampio della madre. ${ }^{29}$ Nella sua teoria evolutiva della coscienza, per certi versi accostabile all'epistemologia genetica di Jean Piaget, Neumann associa le tappe dello sviluppo individuale a quelle della storia della coscienza del genere umano. L'archetipo della Grande Madre, conservativo e antitetico a ogni forma di diffe-renziazione, risulta all'interno di questo processo l'ostacolo maggiore al raggiungimento del pieno sviluppo dell'individuo che, per conquistare la propria parte femminile, deve sviluppare le proprie capacità di separazione e di auto-affermazione.

L'archetipo della Grande Madre è connesso ai culti legati al ciclo di morte e di rinascita delle sementi e alla ciclicità della luna, tipici della civiltà contadina e, ancora più a ritroso, tipici della natura. Non a caso la Grande Madre è simboleggiata, tra

28 Sul tema della Grande Madre sono fondamentali gli studi di Erich Neumann, La Grande Madre. Fenomenologia delle configurazioni femminili dell'inconscio, Astrolabio, Roma 1981; La psicologia del femminile, Astrolabio, Roma 1975 e Storia delle origini della coscienza, Astrolabio, Roma 1978; ma anche quelli di Robert Graves, La Dea Bianca, Adelphi, Milano 1992 e I miti greci, Longanesi, Milano 1955; e quelli più recenti di Marja Alseikaité Gimbutas, Il linguaggio della dea. Mito e culto della dea madre nell'Europa neolitica, Longanesi, Milano 1990 e Le dee viventi, Medusa, Milano 2005; infine il saggio di Roberto La Paglia, La Grande Madre. I culti femminili e la magia lunare, Edizioni Akroamatikos, San Giorgio Jonico 2008.

29 Carl Gustav Jung, L'archetipo della madre. 1939-1954, Bollati Boringhieri, Torino 1981. 
gli altri, sia dal serpente, il cui corpo aderisce alla terra completamente, sia dalle forme circolari, che richiamano questa concezione naturale del tempo. Come osserva Neumann:

La Grande Madre è la Signora del tempo in quanto signora della crescita. La Grande Dea quindi è anche una dea lunare, poiché la luna e il cielo notturno sono le manifestazione evidenti e visibili della temporalità del cosmo, ed è la luna, non il sole, l'autentico cronometro dell'era primordiale. La qualità temporale, così come l'elemento acqua, vanno ascritti al Femminile, la cui natura fluente diviene evidente nel simbolo del flusso del tempo. A partire dalle mestruazioni, sino a giungere alla gravidanza, il Femminile è ascritto al tempo ed è dipendente e determinato da esso più di quanto lo sia il maschile, che tende al superamento del tempo, all'esenzione dal tempo e all'eternità. ${ }^{30}$

Anche Julia Kristeva, da tutt'alto punto di vista, quello semiotico, parla di «un tempo delle donne», ciclico e monumentale, derivato dalla sintesi del tempo della fase patriarcale, storica e cronologica, d'impronta occidentale, giudeo-cristiana e di quello della fase matriarcale, astorica, ciclica e mitica, d'impronta orientale, indù buddista. ${ }^{31}$ Dal punto di vista della critica letteraria, Béatrice Didier nella sua analisi del journal intime come genere letterario ${ }^{32}$ riscontra che l'autobiografia femminile presenta un tempo ciclico, in cui il racconto del sé è il risultato di una composizione corale, quasi di una disposizione circolare delle persone e della materia intorno alla protagonista, la cui identità è data dalla relazione che intercorre con l'altro da sé: è ancora una volta un'identità relazionale. Asma è un testo che condensa questi elementi tipici della narrazione autobiografica femminile: vi si riscontrano gli elementi caratteristici dell'archetipo della Grande Madre elaborato da Erich Neumann, quelli del «tempo delle donne» di Julia Kristeva, quelli della circolarità del journal intime di Béatrice Didier. Ludovica Ripa di Meana di fronte alla necessità di raccontare il rischio della propria morte, per vincere l'angoscia del timore di un nuovo attacco d'asma, crea una serie di disposizioni concentriche, attraverso le quali passa dalla propria morte in atto - la struttura principale del racconto è, infatti, quella del momento in cui la narratrice è vittima della crisi respiratoria - al ricordo della morte della madre; passa da una storia di sé come madre sola con i propri «abbandonati» figli ai ricordi di sé come bambina che ama disperatamente e accudisce con amore tutto materno la bambola della miglior amica; poi, le pagine di diario degli ultimi incontri con Carlo Emilio Gadda e della sua morte, che a prima vista potrebbero apparire quasi una monade interna al testo, giacché non si fa quasi mai menzione del suo dissidio con

30 Erich Neumann, La Grande Madre, cit., pagina 227.

31 Julia Kristeva, "Le temps des femmes", in «Cahiers de recherche des sciences des textes et documents», n. 5, 1979, pagine 5-19.

32 Béatrice Didier, Le journal intime, Presses Universitaires de France, Paris 1976; ma si veda anche il più recente L'écriture femme, Presses Universitaires de France, Paris 1991. 
l'autorità materna: solo alla fine, scolpito, con la definizione potente, con cui l'autrice si congeda dalla sua salma: «il nonamato».

Se un filo c'è, più importante di altri, a cucire tutte queste storie, forse, è quello animato da un discorso matrilineare che, cambiato ciò che va cambiato, ricorda certi pensieri di Virginia Woolf quando invitava le donne, in quanto tali, a pensare attraverso le proprie madri ${ }^{33}$; e quando, mirando alla pace, e invocandola, auspicava «l'arte dei rapporti umani, l'arte di comprendere la vita e la mente degli altri», ${ }^{34}$ ritenendo necessario «trovare nuove parole, inventare nuovi metodi» ${ }^{35}$ per la pace, cioè per la vita. Ciascuna, individualmente, insieme alle altre.

Asma è, dunque, un prendere la parola, un tentativo di modulare la propria voce, $d$ 'individuare il proprio timbro: è un testo per diventare e dirsi scrittrice. E per raggiungere questo obiettivo, Ludovica Ripa di Meana sembra sentire l'esigenza di una specie di regolamento di conti con l'«ordine semiotico della madre» (Julia Kristeva), prima di entrare in un mondo, quello letterario, profondamente segnato dall' «ordine simbolico del padre» (Jacques Lacan). Asma presenta infattiuna ricca e articolata trama di quel linguaggio fatto di segni, carezze, cura affettuosa, legame immediato, che Kristeva ha assegnato all' «ordine semiotico della madre» e Luisa Muraro all' «ordine simbolico della madre». Questi testi non solo presentano un'accentuazione del rapporto madre-figlia, in cui la protagonista ricopre entrambi i ruoli, prima rivolgendosi alla memoria della madre, poi vedendosi come madre dei propri figli; ma mette in scena anche la complessa affettività delle amicizie femminili, declinata nelle varie età della vita.

Anche la dinamica tra interiorità ed esteriorità, tra sé e altro, è segnata da una marca eminentemente femminile all'interno della quale il parallelismo tra respiro e scrittura giunge, alla fine, a prendere la forma, in sogno, di un parto simbolico. Georges DidiHuberman condensando i propri studi sulla filosofia di Pierre Fédida, ${ }^{36}$ in un libro breve quanto intenso, parla dell'aria come veicolo della parola, ma anche quasi come materia organica attraverso cui si articola, si accentua, si respira e si modula il fraseggio della nostra parola. Il dire diviene luogo materiale d'incontro tra soggetto e oggetto, tra significante e significato, e suggerisce una «respiration s'ouvrant à l'autre et signifiant à

33 Virginia Woolf, Una stanza tutta per sé in Romanzi e altro, Mondadori, Milano 1978, pagina 792.

34 Virginia Woolf, Le tre ghinee, Feltrinelli, Milano 1980, pagina 58.

35 Ibidem, pagina 188.

36 Geroges Didi-Huberman, Gestes d'air et de pierre: corps, parole, souffle, image, Les Éditions de Minuit, Paris 2005. 
autrui sa signifiance même». ${ }^{37} \mathrm{Il}$ dire dunque come «témoinage», «pur vocatif», «sincerité», «proximité» all'altro:

«Dire ainsi, [...] c'est s'épuiser à s'exposer, c'est faire signe en se faisant signe sans se reposer dans sa figure même de signe». ${ }^{38}$

Nel suo solito stile affascinante e ricco d'immagini potenti, Georges Didi-Huberman spiega come quest'operazione di conferire aria e gesto al dire si attui in una dinamica di mutuo potenziamento tra parola e silenzio, scambievolmente interni ed esterni:

La plus juste parole n'est sourtout pas celle qui prétend "dire toujours la vérité". Il ne s'agit même pas de la "mi-dire", cette vérité, en se réglant théo-riquement sur le manque structurel dont les mots, par la force de choses, sont marqués. Il s'agit de l'accentuer. De l'éclairer - fugitivement, lacunairement - par istants de risque, décisions sur fond d'indécisions. De lui donner de l'air et du geste. Puis, de laisser sa place nécessaire à l'ombre qui se referme, au fond qui se retourne, à l'indécision qui est encore un décision de l'air. C'est donc une question, une pratique de rythme: souffle, geste, musicalité. C'est donc une respiration. Accentuer les mots pour faire danser les manques et leur donner puissance, consistence de milieu en mouvement. Accentuer les manques pour faire danser les mots et leur donner puissance, consistence de corps en mouvement. ${ }^{39}$

In Asma appunto l'assenza d'aria, di respiro si fa assenza di parola, momento decisivo, sia concretamente perché liminare, sia perché simbolicamente rivelatore:

Se il respiro è luce, io sono il buio. Se il respiro è un pieno, io sono il vuoto. Se il respiro è vita, io sono morte. Un buio, vuoto, morto, non ancora morto. "Se il respiro è voce, io...". Silenzio che si smiagola, emanandosi, nella perfetta cilindricità del laser: è lo sforzo dei visceri, delle natiche, dei polpacci, delle narici; il ronzìo tellurico delle orecchie; la cadenza da reclusorio di unghie, capelli, denti. Ossa. Condannati a esserci. Buio, vuoto, morto, muto. Non ancora. ${ }^{40}$

In questo richiamando alla mente anche il celebre passo del saggio sull'umorismo di Pirandelliano a proposito della forza rivelatrice del silenzio, della sua capacità di dire meglio di tante parole:

In certi momenti di silenzio interiore, in cui l'anima nostra si spoglia di tutte le finzioni abituali e gli occhi nostri diventano più acuti e più penetranti, noi vediamo noi stessi nella vita, e in se stessa la vita, quasi in una nudità arida, inquietante; ci sentiamo assaltare da una strana impressione, come se, in un baleno, ci si chiarisse

37 Pierre Fédida, «Le souffle indistinct de l'image», in La Part de l'œil, n. 9, «Arts plastiques et psychanalyse II», 1993, pagina 220.

38 Ibidem, pagina 223.

39 Georges Didi-Huberman, cit., pagina 9.

40 Ludovica Ripa di Meana, Asma, cit., pagina 2. 
unarealtà diversa da quella che normalmente percepiamo, una realtà vivente oltre la vistaumana, fuori delle forme dell'umana ragione. Lucidissimamente allora la compagine dell'esistenza quotidiana, quasi sospesa nel vuoto di quel silenzio, ci appare priva di senso, priva di scopo, e quella realtà diversa ci appare orrida nella sua crudezza impassibile e misteriosa, poiché tutte le nostre fittizie relazioni consuete di sentimenti e d'immagini si sono scisse e disintegrate in essa. Il vuoto interno si allarga, varca i limiti del nostro corpo, diventa vuoto intorno a noi, un vuoto strano, come un arresto del tempo e della vita, come se il nostro silenzio interiore si sprofondasse negli abissi del mistero. ${ }^{41}$

Ma Asma nasce, in principio, come la storia di una vicenda incentrata sulla minaccia della morte in cui resta centrale il corpo nel momento in cui vien meno il respiro, ovvero nel momento in cui si acutizza la percezione. In questo, come suggerisce Paul Shilder giocano un ruolo fondamentale gli orifizi, che sono sì luoghi del confine tra esterno e interno, tra sé e mondo, ma solo in apparenza, essendo invece questa consapevolezza affidata alla nostra percezione, che avviene sempre qualche centrimetro in dietro rispetto alla superficie:

Les points les plus importants du corps en sont les orifices, et, naturellement, il sont le siège de sensations très particulières. Quand nous respirons en fermant la bouche, nous éprouvons des sensations particulières dans le nez; mais aussi quand nous respirons bouche ouverte et que nous ne sommes pas conscients de repsirer, et même quand nous arrêtons de respirer, nous sentons distinctement l'intérieur des narines. Ce qui est important, c'est que nous les sentons près de l'orifice, non pas vraiment au bord de narines mais à environ un centimètre en retrait. À ce niveau, nous sentons ou bien quelque chose de spécifique, ou bien la fraîcheur de l'air. Il en va de même pour la bouche. Nous ne sentons pas notre bouche véritablement au bord des lèvres. La zone sensible est, là encore, à environ un centimètre en retrait. Quand nous respirons par la bouche, nous sentons l'air sur la voûte du palais mais il semble que nous le sentions aussi dans le tiers antèrieur de la cavité buccale. Si nous respirons très profondément, nous sentons l'air au fond de la bouche et même dans la région du sternum: mais pas plus bas que la pointe du sternum et pas plus avant qu'à un ou deux centimètres de la surface. Nous pouvons dire d'une manière générale que les zones les plus sensibles du corps sont situées près des orifices, mais à un ou deux centimètres en retrait de la surface. ${ }^{42}$

In tutte le sequenze della cornice di Asma, quelle in prima persona, al tempo presente, in cui si racconta appunto della crisi respiratoria avvenuta nella stanza del

41 Luigi Pirandello, L'umorismo, a cura di Daniela Marcheschi, Mondadori, Milano 2011.

42 Paul Schilder, L'Image du corps. Ètude des forces constructives de la psyché, Gallimard, Paris 1968, pagine 12-13. 
Grand Hôtel di Vienne, nella Valle del Rodano, appare come costante il passaggio dalla percezione corporale, esasperata dalla gravità della situazione, all'esterno della quotidianità e all'esterno della memoria, del passato.

Soffoco.

Gli occhi roteano da un punto all'altro del loro abitacolo. Le iridi si slanciano verso l'alto inficcandosi sottole palpebre superiori o, in diagonale, verso la tempia e la radice del naso, esibendo due mezze sclerotiche da martire, due bianche mezze lune orlate dal taglio bagnato e rosa della palpebra inferiore. Vorticano e vertiginano. Maelström del respiro. Amen, pazzo, irresponsabile, deflagra, atomizzando i secondi del proprio tempo, il boato del telefono. La voce risponde pianissimo, a monosillabi timbrati, disperati: oui, non, pas encore? essayons, et le pompiers? vit... trac. Cornetta riappesa. Microfono appoggiato con cautela. Gesti officiati in punta di gesto. Il laser del pipistrello morente si è cavernizzato in un rantolo assordante: da licantropo col raffreddore, da fantasma che riattiva una segheria abbandonata, e questa volta si è deciso: ci dà dentro. I capelli... i capelli... aiuto, sono diventati di marmo, pesano sulla nuca, non resisto, il pettinino... dov'è per tirarli su, come faccio a dirlo?, aiuto, ora schiacciano il sommo della testa, intorno alle orecchie stringono, la spaccheranno, vedrai, e il cervello, spiaccicato, li appiccicherà tutti, in ciuffi, poi vàlli a spicciare, con la spazzola, vedrai che rogna e che male, si strapperanno ciocca per ciocca, e rimarrò calva. Chi mi salva? ${ }^{43}$

Fondamentale, come s'è accennato sopra, il collegamento tra la propria morte e quella della propria madre, che è apparentemente affidato alla banale invocazione del linguaggio quotidiano («mamma, come si soffre...») e invece è decisivo.

Vecchia. Ora,morta,enonancora.Nonancora.Chepeccato.Nonpossospiegarenientea nessuno egliocchi... ecco, stanno per schizzare fuori, li sento sgusciare via dalle palpebre come lupini dalla buccia, ma, possibile?... il bulbo dovrebbe far capo a un fascio di nervi, muscoletti, filamenti che lo ancorano dentro l'orbita... mamma, come si soffre a morire... Ti ho vista la fatica che hai fatto, povera mammetta - altroché - in quei due giorni, uno passato a occhi sempre aperti, l'altro con gli occhi sempre chiusi, sempre nella stessa posizione, e non finivano proprio mai, soprattutto il profilo era sbalordito, la bocca aperta in controluce sembrava un'amaca vuota, ma di faccia, quando mi alzavo in piedi per guardarti meglio, per guardarti tutta, eri proprio spiccicata l'Eva di Masaccio, il nero disco di un'eclissi al posto della bocca. ${ }^{44}$

«L'Eva di Masaccio» è l'unico riferimento diretto presente in questo testo a un'immagine dipinta: l'autrice ha smesso di respirare, è sul punto di morire, e in una

43 Asma, cit., pagine 6-7.

44 Ibidem, pagine 8-9. 
sorta di parallelismo rievoca l'immagine della bocca della madre morta, spalancata sul buio («il nero disco di un'eclissi»). Poco più avanti, la scrittrice constata che non avrebbe mai pensato che «il terrore potesse somigliare così tantoalla pittura», rivelando quella che si dimostrerà essere nei suoi testi successivila sua vocazione principale: la capacità di conferire ai propri versi una potenzavisionaria notevole. Specie nella rappresentazione della «violenza della realtà», per dirla con le parole del pittore Francis Bacon, molto amato anche da Giovanni Testori: non a caso, infatti, l'espressionismo di Ludovica Ripa di Meana può essere a buon diritto accostato a quello del narratore, drammaturgo e poeta lombardo.Una visionarietà, quella testoriana, che mi sento di evocare a maggior ragione, alla luce del brano seguente, dove la resa dei conti con la propria morte in parallelo alla resa dei conti con la morte della propria madre diventa il momento di riconoscimento del proprio daimon, che appunto per i greci stava alle spalle della persona, risultandole invisibile. Un daimon che si confronta con l'immagine di una madre-Dio, «più a portata di mano e meno complice» di Dio:

Ma quel che non riesco a perdonarmi, neanche ora che sto morendo io, è di averti guardata, addirittura scrutata, nel primo dei due giorni, quello che ti sei campato per intero con gli occhi tutti sbarrati. Non avrei mai pensato che il terrore potesse somigliare così tanto alla pittura, potesse roteare così irredento dentro due occhi fissi. Io non dovevo osare, con il mio sguardo. Né gli altri, avrebbero dovuto. Bisognava tirare cortine intorno a te che rendessero inviolabile il vitreo panico dei tuoi famosi occhi azzurri, la indecente staticità della tua sofferenza. Eri sacra, remota, non come adesso che sei morta da quasi quattro anni e che ti sfruttocome invocazione domestica nella mia agonia... è vero che ti intercalo con Dio, matu sei più a portata di mano e meno complice... La tua irraggiungibilità metteva terrore, non c'entrava un bel niente con quel caos di esperienze irritante e consuetoche consideriamo essere proprio la vita... tu respiravi da morta, guardavi da morta, tacevi da morta... imperturbabile come una morta, eri viva. Premonizione capovolta, eri già il sogno che avrei sognato, ma accarnato lì, un sogno da odorare, da controllare, da toccare e, soprattutto, da non lasciar svanire... madre troppo grassa, madre troppo magra, cicciona, scheletrica, ricca-ricca, povera-povera, madre sempre troppo... autoritaria, umiliata, dura, delicata, fredda, sensuale, indiscreta, intirizzita. Temeraria, intelligente. Con le tue perle, anche tu. ${ }^{45}$

Il congedo dalla madre, che diventa memoria e dunque appropriazione avviene, ancora una volta, attraverso un dettaglio della quotidianità, «Con le tue perle, anche tu.», che è il segno apparentemente superficiale del riconoscimento di una genealogia, non solo e non tanto nel senso di un'ereditarietà e di una tradizione, quanto piuttosto nel senso, tutto simbolico, del riconoscimento identitario, di un sé necessariamente relazionale, che non esiste senza l'altro.E, infatti, è alla madre che l'autrice chiede di raccontare la propria infanzia, riproponendoil processo edipico per cui la propria storia è inconoscibile se non raccontata da un altro:

45 Ibidem, pagine 9-10. 
Ma mi dici com'era la bambina, non mi ricordo più niente... che faceva, dove? - Mi indicate il niente. Ci vado ricca. Per escluderlo. ${ }^{46}$

Seguono una serie di capoversi, di circa una pagina ciascuno, ${ }^{47}$ quasi lasse di un poemetto di ricordi che affiorano dall'infanzia, cuciti da un incipit anaforico, ennesimo segnale di un'evidente tendenza del testo a una dimensione performativa, orale, tipica della poesia popolare:

«La bambina si sdraiò, pancia a terra...»;

«La bambina voleva volare...»;

«La bambina aveva chiuso gli occhi, strizzan-doli forte...»;

«La bambina lì per lì si era offesa...»,

«La bambina era rimasta immobile, i pugni stretti nelle tasche...»;

fino all'ultimo, che, riproponendo ancora una volta una struttura circolare, va a terminare da dove aveva cominciato: «Quella notte la bambina sognò sua madre...».

Poi, una sequenza ospedaliera ${ }^{48}$, che ritorna al presente, all' inizio del testo, alla crisi di asma. Anche qui l'incipit dei capoversi è segnato in maniera forte:

«Prima: un corpo grande, posato a casaccio sul lettino...»;

«Poi: verso le sette del mattino...»;

«Poi: verso le nove, nel corridoio...»;

«Poi: verso le undici, nella terapia intensiva...»;

«Poi: alle quattro del pomeriggio, come una malata di mente...»;

«Poi: alle otto di sera, appoggiata ai cuscini...»

per concludersi con un capoverso dall'andamento decisamente elegiaco, in cui la musicalità della frase riconduce al clima dell'immagine infantile finale:

Sulla striscia di asfalto, bordata di stoppie e cespugli di salvia, ormai lontani nel tronco di cono che si allunga sempre di più nel retrovisore, dopo tre giorni un padre infelice vestito di bianco, mi saluta. Accanto a una figlia, infelice, vestita di rosa come quando era bambina. ${ }^{49}$

La conclusione della scena con il saluto della figlia alla narratrice, che la vede andarsene vestita del colore con lui la vestiva da bambina,è la scintilla per incastrare, in questa struttura di richiami figlia-madre, l'altra struttura madre-figlia: in cui la narratrice da figlia diventa madre. La sequenza successiva, infatti, mette in scena il dialogo tra la narratrice e il suo lettore,ricalcando quello che possiamo ipotizzare essere stato un dialogo tra l'autrice e il marito, che sappiamo lettore fin dalle prime pagine di questo testo.

46 Ibidem, pagine 11.

47 Ibidem, pagine 11-17.

48 Ibidem, pagine 17-19.

49 Ibidem, pagine 19. 
- Ma come? Non fai finire la bambina volante? ... - Non lo so. Non ora, ora no. Ora voglio ricordare la MIA bambina, la mia abbandonata bambina, e il suo abbandonato FRATELLO, i miei due abbandona-tissimi figli... - Ma quando mai? Ti confondi, hai abbandonato il padre... - ... e tutto per inseguire l'amore, l'amore assoluto... - Cielo, che enfasi! Sì, ma sempre con loro appresso, lo inseguivi, per modo che quei poveri amanti se ne trovavano tre alla volta sul groppone! Non usare questo tono, proprio ora... - Ti do una mano per non affogare in un paté di pathos: morendo, posso capirti, la tentazione è grande. - Abbandonati, abbandonatissimi, straabbandonatissi-mi... - E piantala! Non ti ricordi quando il padre te li ha presi e tu, per riaverli, un biennio di avvocati, una figlia nuova, sua di lui, con un'altra, e tu, che un altro po' e ti partiva il cervello... non ti ricordi, no? - Esageri... - Vorrai dire che esagera il diario... (Tippettandosi il lobo destro) Come fa? Come fa? ${ }^{50}$

Seguono, infatti, pagine di diario che ricostruiscono gli ultimi giorni delfebbraio 1962, trascorsi a Genova. ${ }^{51}$ Ma sono le date stesse (28 febbraio, mapoi 29, 30, 31 e 32 febbraio) a dichiarare una scrittura a posteriori, in cui l'eventuale presenza di un testo originario, storico, cede il passo alla sua riscrittura, alla voce che racconta ora i sentimenti materni della narratrice.A queste aperture di memoria si contrappone la chiusura del corpo che non respira, lasituazione di stallo delle funzioni vitali che precede la morte, come a sottolineare per contrasto la vitalità dei ricordi, della consapevolezza della propria vita.

Sto morendo. Sto morendo. Sto morendo. Sto morendo. Sto morendo. Sto morendo. Sto morendo. Sto morendo. Sto morendo.

Sto morendo. Sto morendo. Sto morendo. Sto morendo. Sto morendo. Sto morendo. Sto morendo. Sto morendo. Sto morendo.

Sto morendo. Sto morendo. Sto morendo. Sto morendo. Sto morendo.

Nella tomba i muri son fatti così. Fanno casetta. Soffoco. Non riesco a strapparmi via lo sterno, è lui l'osso che mi si è conficcato in petto fin dalla nascita. Le mani a furia di raspare riusciranno e raggiungere la guaina del pettorale e la acchiapperanno,la scheggia maledetta che mi sta ammazzando. Non è lo sterno. Non è lo sterno, e lo sterno non è una scheggia. È asma, solo asma, asma pura, asma distillata, asma tutta per te. Aiutami, ninno, aiutami, la luna... ora il sangue sprizza dalle orecchie, dalle dita dei piedi, divento fontana, anche dall'ombelico, dal culo, no, è tutto imballato, non esce niente. Nemmeno il respiro. Dio. ${ }^{52}$

50 Pagine 19-20.

51 Pagine 19-20.

52 Ibidem, pagine 35. 
Questo contrasto si fa evidentissimo quando, come nella sequenza che segue, Ludovica Ripa di Meana arriva a cogliere nell'essenza del rapporto con la propria madre («quel paso doble, dall'inizio della vita») l'essenza della propria personalità: il proprio daimon, appunto.

La bambina afferrò con la sua sinistra, la mano destra della madre che stava guidando, e non la mollò più. Aveva tre anni, un vestito blu a puntini bianchi e una fascia di filanca che le teneva indietro, stempiandola, i fini capelli lisci e corti. Non parlava, né si voltava. La fronte alta e leggermente bombata disegnava il promontorio di una sua concentrazione criminale. Guardava davanti a sé, all'altezza del cruscotto, e lasciava penzolare i piedi nel vuoto con calma ferocia. E calma e feroce strizzava la mano della madre, soprattutto quando la sentiva svincolarsi nei gesti della guida. L'altra cominciava a percepire la mano tonda della figlia come un'escrescenza nata all'interno della propria grande mano asciutta, un raddoppio miniaturizzato e un po' mostruoso e, arresa, le muoveva velocemente all'unisono, quella mano e quella manina, intorno al volante, sul cambio, sulle frecce, convinta ormai che avere le mani fosse sempre stato così, quel paso doble, dall'inizio della vita. Quando la ottoecinquanta si fermò all'ombra, sotto la casa di suo padre, la bambina cominciò a strofinarsi il dorso della mano materna contro la guancia piano piano con moto circolare, gli occhi perduti su un'asta sciancata del tergicristallo (l'altra giaceva a destra perfettamente svenuta) che, azionato per sbaglio, ruggiva sul vetro calcinato dal sole di luglio, mentre di profilo la madre, conteso con uno strattone alla figlia quell'involtino di mani, ci si asciugava una biglia di lacrima proprio alla radice del naso. Si tenevano. Non si guardavano. Di profilo. Tassative. Con il loro amore tremendo. Così, più o meno, andavano le cose, intorno alle quindici, in un giorno del millenovecentocinquantanove, appunto, di luglio. Per dire. Di una inconsolabile bambina. Anzi, di due. ${ }^{53}$

Per rendere l'eterogeneità con cui è composto questo testo, Asma, e la difficoltà di attribuzione a un genere letterario che non sia la scrittura privata, autobiografica, basti rilevare come a questa sequenza ne segue un'altra intitolata Lui la mamma l'altro, ${ }^{54}$ vero e proprio testo teatrale, un breve atto unico che si conclude con una filastrocca per bambini.

Poi «un ricordo roseo, pasquale, di papà, ${ }^{55}$ piuttosto breve, e quindi Storia di En, in terza persona, ennesima variazione autobiografica:

...sapeva che le sue vite erano li, con lei, non tessute insieme ma accostate, una accanto all'altra, ognuna col suo mistero solitario di oggetto. En entrava in una nuova vita come in una casa, e chiudeva la porta. Posava intorno a sé i segni

53 Ibidem, pagine 36-37.

54 Ibidem, pagine 38-48.

55 Ibidem, pagine 49-53. 
della sua solitudine precedente, e penetrava con una passione disperata in quella, sconosciuta, che le stava davanti. Fino a quando non avesse avuto la rivelazione più remota di quanto inesauribile fosse la sua solitudine, En non sarebbe più uscita da quella casa che lei, parafrasando, chiamava amore. ${ }^{56}$

Poi, un sogno di morte ${ }^{57}$ a precedere le pagine di diario che raccontano gli ultimi incontri e la morte di $\mathrm{G}^{* 58}$, chiaramente identificabile con lo scrittore Carlo Emilio Gadda, che sono oggetto del capitolo seguente, «Il nodo alla cravatta».

La Storia di En riprende descrivendo un circolo tutto femminile intorno al quale nasce e si sviluppa l'amicizia tra due bambine. Ancora una volta la storia prende l'avvio con una successione di capoversi che procedono come lasse di un poema, fortemente segnati nell'incipit dall'evocazione del personaggio di cui si parla:

«En. La sua migliore amica aveva i capelli rossi e una bambola di celluloide e gomma che si chiamava Flavia...«»

«En amava morbosamente Flavia. Un po' meno, la sua migliore amica...»

«La cugina della sua miglior amica era loro coetanea e passava anche lei per migliore amica...»

«La sorella più grande, in effetti, era bella davvero, a dodici anni era bella come una sposa del deserto...»

«Del fratello, c'era poco da dire...»

«La madre, festeggiata dagli anziani genitori azkhenazi e dalla sua unica sorella

$[\ldots]$ come una gran bellezza...»

Una narrazione che procede per personaggi, che sono sempre, nei testi di Luvodica Ripa di Meana, persone, di cui si coglie la fisicità, la psicologia, l'estrazione sociale, la realtà.

Questa struttura narrativa, imperniata intorno a un personaggio, rappresenta un'inclinazione, un punto di vista, quasi uno stilema narratologico di Ludovica Ripa di Meana, facilmente riconoscibile nella maggior parte dei suoi testi, come dimostrerò nel capitolo nono, intitolato «Soggetto».

La storia delle bambine e della bambola Flavia s'inter-rompe con l'allontanamento dovuto ai casi della vita. En e l'amica si ritrovano, a distanza di anni, quando quest'ultima la convoca in punto di morte.

Quindi il testo torna al presente e al ricordo della madre che appare all'autrice come fantasma ${ }^{59}$ in un'allucinazione, in pieno giorno, mentre è alla finestra, piangendola, poco dopo la sua morte.

56 Ibidem, pagine 55.

57 Ibidem, pagine 59.

58 Ibidem, pagine 59-70.

59 Ibidem, pagine 82-84. 
Di nuovo, la Storia di En prende voce e si riempie di memorie della giovinezza, ancora una volta con tutta evidenza autobiografiche.

Infine, il presente, l'asma, in una sequenza estremamente significativa dal punto di vista simbolico, in cui alla crisi respiratoria si sovrappone la vocazione alla scrittura: l'idea del recupero del respiro è rappresentata come la necessità di urlare (che è anche gridare, gridare il proprio dolore, e quindi per traslato cantarlo, raccontarlo e raccontarselo, dunque scriverlo).

Urla. Ora urla. Urla qui. Proprio. Una sirena urla. Mi urla. Nel petto, in testa, urla, attraverso. Verso. Il porto. Lì. Urla. Sirenaurla. Oh, Sire. Oh. Sire, sirena, sirenetta, sirenella, chiamarsi almeno Serenella, un po' come loro, le stupende misteriose, le ondulanti capigliate di blu, che salgono lente e poi, con un sorso, s'inghiottono da sé. Oh, allungare da sott'acqua tesori ai marinai, acqua in bocca volare negli abissi, far buchi nell'acqua per tenersi a galla, aver l'acqua alla gola per innamorare e i mozzi e i galeotti e i re. Oh, essere sirena, Sire, per una volta sola, i freddi seni crudi di nuovo, le spalle belle e l'arco della schiena inarcato in viola come lo spicchio della luna dentro un night. Ma allora perché urli, sirena sirenetta nemmeno Serenella, e squarci di fatica il soffio che non abbiamo più? Urli perché sei chiusa, urli perché sei serrata, combaciata, segregata, urli perché sei interrotta, inclusa, insaccata. Urli perché sei impesciata. Urli, senza la fica. Urla, la fica, d'asma. ${ }^{60}$

L'immagine finale, liberatoria e rivelatrice, coincide con quella dell'antica divinità Baubo, ${ }^{61}$ riconducibile all'archetipo della Grande Madre. Baubo è priva del capo, come d'altro canto appaiono mutile degli arti molte divinità primitive femminili, legate alla terra e alla sfera della fecondità. Baubo parla tramite la vagina, evocando simbolicamente un parlare fatto di materia prima, un parlare vitale che trae origine dalla propria natura e dalla natura in genere; e, infatti, a questa dea primitiva si è sovrapposta la figura di Iambe, a cui - con erronea, ma felice etimologia - si fa risalire l'origine del verso caratteristico delle parti dialogate della tragedia, il giambo.

In questa sequenza si condensano molti elementi inte-ressanti e rivelatori della scrittura di Ludovica Ripa di Meana: l'immagine iniziale delle sirene, ad esempio, è caratterizzata da un giocare con le parole, affidandosi al loro suono,inseguendo il fascino della loro musica, tipico dell'infanzia e, come ha ben evidenziato Julia Kristeva - e come spiegherò meglio in seguito - appartiene all'«ordine semiotico della madre». In più, come a sottolineare la necessità di un parola fertile, naturale (nel senso di primigenia) e quindi veritiera, che è poi anche un evocare la scelta di una letteratura necessaria, al canto delle sirene, seducente quando sterile, si sostituisce quello della fica - Baubo, osceno quanto fruttifero.Il tempo di un'altra storia, di cui è protagonista

60 Ibidem, pagine 94.

61 Si vedano in proposito i riferimenti bibliografici della nota 14 in questo stesso capitolo. 
ancora una volta una ragazzina e di cui la narratrice pare essere solo una testimone, e siamo alla sequenza finale, dominatadall'im-magine di un parto simbolico:

Perché scricchiola il mondo? E cos'è questo rombo totale? Ecco che si volta... si volta... La clessidra ${ }^{62}$ ora è capovolta su sé: cola la sabbia tutta uguale, e mi riempie. Mi scoppia il cuore. Chi preme ora? io, devo uscire, tutto stringe, si stringe, forza signora, spinga, spinga - allora sono loro, tutti e due insieme, spingo, spingo, che buio però, cos'è ora, dove sono finita, in una nassa, no, un cunicolo, che più mi muovo più mi sento stringere, i cerchi concentrici che mi premono il petto contro la schiena, me li incollano insieme, cosa sono? aiuto, lo so, l'hoola-hop del demonio che arriva verticale dall'inferno, lo riconosco, l'ho riconosciuto, gira, cinge, strina, brucia sempre più veloce, mi stritola, ${ }^{63}$ ecco, stritolata, sfarinata, incenerita, dissolta, ah, meglio ora, il cuore bussa lontano, potrò dormire, i cerchi sono tornati costole, posso alzarmi, leggera, vuota, né carne, né pelle, né occhi, né bronchi, solo ossa più bianche della luna, solo sterno, e costole snelle piene di vento. Un vento silente. Immobile. Un ventoche En poteva volare. ${ }^{64}$

Il richiamo al «vento che En poteva volare» che si sovrappone e si confonde con il riconquistato respiro riconduce il lettore a una delle prime sequenze del testo, in cui la bambina aveva sognato di poter volare e, con ennesimo gesto circolare, le ultime righe di Asma tornano da dove il testo aveva preso l'avvio:

Francia, Valle del Rodano, Vienne, Grand Hôtel du Nord, terzo piano, stanza 308.

Bussano. Entra Kiledjian Eric, 01 - Omnipraticien Conventionné. ${ }^{65}$

\section{BIBLIOGRAFIA}

62 «Che accadrebbe se un giorno o una notte, un demone strisciasse furtivo nella più solitaria delle tue solitudini e ti dicesse: "Questa vita, come tu ora la vivi e l'hai vissuta, dovrai viverla ancora una volta e ancora innumerevoli volte, e non ci sarà in essa mai niente di nuovo, ma ogni dolore e ogni piacere e ogni pensiero e sospiro, e ogni indicibilmente piccola e grande cosa della tua vita dovrà fare ritorno a te, e tutte nella stessa sequenza e successione [...]. L'eterna clessidra dell'esistenza viene sempre di nuovo capovolta e tu con essa, granello della polvere!". Non ti rovesceresti a terra, digrignando i denti e maledicendo il demone che così ha parlato? Oppure hai forse vissuto una volta un attimo immenso, in cui questa sarebbe stata la tua risposta: "Tu sei un dio e mai intesi cosa più divina"?» Friedrich Nietzsche, aforisma 341, in La gaia scienza e Idilli di Messina, Adelphi, Milano 2005.

63 «Così discesi del cerchi primaio / giù nel secondo, che men loco cinghia / e tanto più dolor, che punge a guaio. / Stavvi Minòs orribilmente, e ringhia: / essamina le colpe ne l'intrata; / giudica e manda secondo ch'avvinghia. / Dico che quando l'anima mal nata / li vien dinanzi, tutta si confessa: e quel conoscitor de le peccata / vede qual loco d'inferno è da essa; cignesi con la coda tante volte / quantunque gradi vuol che giù sia messa. / Sempre dinanzi a lui ne stanno molte: / vanno a vicenda ciascuna al giudizio, / dicono e odono e poi son giù volte.» Dante Alighieri, Inferno, V, vv. 1-15.

64 Asma, cit., pagina 98.

65 Ibidem, cit., pagina 99 
Arendt, A. (1987). La vita della mente, Il Mulino: Bologna.

Arendt, A. (1989) Vita activa. Bompiani: Milano.

Arriaga Flórez, M. (1997). Mio amore, mio giudice. Alterità autobiografica femminile. Manni: Lecce.

Bachtin, M. (1979) Estetica e romanzo. Einaudi: Torino.

Bachtin, M. (2001) Estetica e romanzo. Enaudi: Torino.

Cavarero,A. (2001). Tu che mi guardi, tu che mi racconti. Filosofia della narrazione, Feltrinelli: Milano.

Celentano, A. (1982), Il paradiso è un cavallo bianco che non suda mai. Sperling \& Kupfer: Milano.

Derrida, J. (1993) Otobiographies. L'insegnamento di Nietzche e la politica del nome proprio, Il Poligrafo, Padova.

Fédida, P. (199) Le souffle indistinct del'imange, La part del'oeil, Arts plastique setpsychanalyse II.

Ferrari, F. (1987) Edipo re, Edipo a Colono. Rizzoli: Milano

Foucault, M. (1972) L'ordine del discorso. Einaudi: Torino.

Gimbatus, M. (2005). Le dee viventi. Medusa: Milano

Gimbutas, M. A. (1990). Il linguaggio della dea. Mito e cultodella dea madre nell'Europa neolitica. Longanesi: Milano

Graves, R. (1955) I miti greci, Longanesi, Milano.

Graves, R. (1992) La Dea Bianca, Adelphi, Milano.Guglielminetti, M. (1986) Biografia e autobiografia, in Letteratura italiana, V: Le Questioni, Einaudi, Torino.

Huberman, G. (2005). Gestesd'airetdepierre:corps, parole, souffle, image. Les Éditions de Minuit: Paris

Jung, C. G. (1981) L'archetipo della madre. Bollati Boringhieri: Torino.

La Paglia, R. (2008). La Grande Madre. I culti femminili e la magia lunare. Edizioni Akroamatikos: San Giorgio Jonico

Neumann, E. (1975) La psicologia del femminile. Astrolabio: Roma.

Neumann, E. (1978) Storia delle origini della coscienza. Astrolabio: Roma.

Neumann, E. (1981) La Grande Madre.Fenomenologia delle configurazioni femminili dell'inconscio. Astrolabio: Roma.

Nietzsche, F. (1986). Ecce homo. Come si diventa ciò che si è. Adelphi: Milano.

Pirandello, L. (2011). L'umorismo, a cura di Daniela Marcheschi. Mondadori: Milano

Raimondi, E. (2018). Camminare nel tempo. Una conversazione con Alberto Bertoni e Giorgio Zanetti. Quaderns d'Italià, 23, 201-204. 
Ripa di Meana, L. (1989) Asma, inedito

Ripa di Meana, L. Contini, G. (1989) Diligenza e voluttà. Longanesi: Milano.

Schilder, P. (1968) L'Image du corps.Ėtude des forces constructives dela psyche. Gallimard: Paris

Villanueva, D. (1992). Realidad y ficción: la paradoja de la autobiografía, in Escritura autobiográfica,José Romera Castillo e altri (a cura di), Actas del seminario internacional del Instituto de Semiótica literaria y teatral,Madrid, UNED,1-3 luglio,Visor, Madrid.

Zambrano, M. (1992). I beati. Feltrinelli: Milano.

Zeri, F. (1987). Dietro l'immagine. Conversazioni sull'arte di leggere l'arte. Longanesi: Milano. 